\title{
Coulomb Gap and Correlated Vortex Pinning in Superconductors
}

\section{Citation}

Täuber, Uwe C., Hongjie Dai, David R. Nelson, and Charles M. Lieber. 1995. “Coulomb Gap and Correlated Vortex Pinning in Superconductors." Physical Review Letters 74 (25): 5132-35. https://doi.org/10.1103/physrevlett.74.5132.

\section{Permanent link}

http://nrs.harvard.edu/urn-3:HUL.InstRepos:41417267

\section{Terms of Use}

This article was downloaded from Harvard University's DASH repository, and is made available under the terms and conditions applicable to Other Posted Material, as set forth at http:// nrs.harvard.edu/urn-3:HUL.InstRepos:dash.current.terms-of-use\#LAA

\section{Share Your Story}

The Harvard community has made this article openly available.

Please share how this access benefits you. Submit a story.

Accessibility 


\title{
Coulomb Gap and Correlated Vortex Pinning in
}

\section{Superconductors}

\author{
Uwe C. Täuber, ${ }^{1}$ Hongjie Dai, ${ }^{2}$ David R. Nelson, ${ }^{1}$ and Charles M. Lieber ${ }^{2}$ \\ ${ }^{1}$ Lyman Laboratory of Physics, Harvard University, Cambridge, Massachusetts 02138 \\ ${ }^{2}$ Division of Applied Sciences and Department of Chemistry, Harvard University, Cambridge, \\ Massachusetts 02138
}

(February 1, 2008)

\begin{abstract}
The positions of columnar pins and magnetic flux lines determined from a decoration experiment on BSCCO were used to calculate the single-particle density of states at low temperatures in the Bose glass phase. A wide Coulomb gap is found, with gap exponent $s \approx 1.2$, as a result of the long-range interaction between the vortices. As a consequence, the variable-range hopping transport of flux lines is considerably reduced with respect to the noninteracting case, the effective Mott exponent being enhanced from $p_{0}=1 / 3$ to $p_{\text {eff }} \approx 0.5$ for this specific experiment.
\end{abstract}

PACS numbers: 74.60.Ge, 05.60.+w

Typeset using REVTEX 
The remarkably rich phase diagram of magnetic flux lines in high-temperature superconductors, especially when subject to point and/or extended pinning centers, has attracted considerable experimental and theoretical interest [1]. Understanding the interaction of vortices with defects is especially important since flux lines must be pinned to mimimize dissipative losses from flux creep. A promising pinning strategy involves the creation of linear damage tracks in materials by heavy ion irradiation. These columnar defects have been found to increase the critical current, as well as to shift the irreversibility line significantly upwards [2].

A theory of flux pinning by correlated disorder has been developed to explain these results, exploiting a formal mapping of the statistical mechanics of directed lines onto the quantum mechanics of two-dimensional bosons [3]. In this study, the intervortex repulsion, whose range is determined by the London penetration depth $\lambda$, was only treated using approximate, order of magnitude estimates. However, if $\lambda \gg a_{0}$, where $a_{0}=(4 / 3)^{1 / 4}\left(\phi_{0} / B\right)^{1 / 2}$ is the average distance between vortices ( $\phi_{0}=h c / 2 e$ is the elementary flux quantum), the interactions become effectively long-range, and may lead to important correlation effects.

Indeed, the analogy of flux lines at low temperatures pinned to columnar defects (Bose glass phase) with two-dimensional localized carriers in doped semiconductors (Coulomb glass) [4, suggests that a "Coulomb" gap should emerge in the single-particle density of states (distribution of vortex pinning energies). Because such a gap will affect significantly the current-voltage characteristics in a variable range hopping approach [3], it is important to estimate the size of the Coulomb gap in the Bose glass phase and to understand the correlation effects induced by the intervortex repulsion. The recent successes, moreover, in simultaneously measuring both the columnar defect and flux line positions [0, [0 allow for detailed comparison of the spatial correlations found in experiment and theory. Such a comparison is not feasible in the semiconductor case.

The density of states may be obtained by using a variant of the Monte Carlo algorithm described by Shklovskii and Efros [⿴囗十. [7]. Using the experimentally determined columnar defect and flux line positions shown in Fig. 1, we can predict the density of states and the 
transport characteristics for this specific sample in the variable range hopping regime, at temperatures slightly below the depinning transition. We find that the ensuing Coulomb gap is remarkably large, even in the case $\lambda \approx a_{0}$, i.e. when the interactions are relatively short-range. Vortex interactions raise the effective Mott exponent from the non-interacting result $p_{0}=1 / 3$ to a value $p_{\text {eff }} \approx 0.5$ for this specific sample. These results imply that correlation effects strongly enhance the pinning of flux lines to columnar defects.

We consider the following model free energy for $N$ flux lines, defined by their trajectories $\mathbf{r}_{i}(z)$ as they traverse the sample of thickness $L$, with the magnetic field $\mathbf{B}$ aligned along the $z$ axis (perpendicular to the $\mathrm{CuO}$ planes), [3]

$$
\begin{gathered}
F=\int_{0}^{L} d z \sum_{i=1}^{N}\left\{\frac{\tilde{\epsilon}_{1}}{2}\left(\frac{d \mathbf{r}_{i}(z)}{d z}\right)^{2}+\frac{1}{2} \sum_{j \neq i}^{N} V\left[r_{i j}(z)\right]\right. \\
\left.+\sum_{k=1}^{N_{D}} V_{D}\left[\mathbf{r}_{i}(z)-\mathbf{R}_{k}\right]\right\}
\end{gathered}
$$

Here $r_{i j}(z)=\left|\mathbf{r}_{i}(z)-\mathbf{r}_{j}(z)\right|$, and $V(r)=2 \epsilon_{0} K_{0}(r / \lambda)$ is the repulsive interaction potential between the lines; the modified Bessel function $K_{0}(x) \propto-\ln x$ for $x \rightarrow 0$, and $\propto x^{-1 / 2} e^{-x}$ for $x \rightarrow \infty$. Thus the (in-plane) London penetration depth $\lambda$ defines the interaction range. The energy scale is set by $\epsilon_{0}=\left(\phi_{0} / 4 \pi \lambda\right)^{2}$, and $\tilde{\epsilon}_{1}$ is the tilt modulus. Finally, the pinning energy is a sum of $N_{D} z$-independent potential wells $V_{D}$ with average spacing $d$ centered on the $\left\{\mathbf{R}_{k}\right\}$, whose diameters are typically $c_{0} \approx 100 \AA$, with a variation induced by the dispersion of the ion beam of $\delta c_{k} / c_{0} \approx 15 \%$. This induces some probability distribution $P$ of the pinning energies $U_{k}$, which may be determined from the (interpolation) formula $U_{k} \approx\left(\epsilon_{0} / 2\right) \ln \left[1+\left(c_{k} / \sqrt{2} \xi\right)^{2}\right]$, where $\xi$ is the coherence length [3]. E.g., for $\lambda \approx 4200 \AA$ one has $U_{0} \approx 0.67 \epsilon_{0}$, and $w=\sqrt{\left\langle\delta U_{k}^{2}\right\rangle} \approx 0.1 \epsilon_{0}$.

As is explained in detail in Ref. [3], the statistical mechanics of the model (II) can be formally mapped onto a two-dimensional zero-temperature quantum mechanical problem by using a transfer matrix approach. In this boson analogy, the real temperature $T$ assumes the role of $\hbar$ in the quantum problem, and the boson electric field and current density map on the superconducting current $J$ and the true electric field $\mathcal{E}$, respectively (see Table I in 
Ref. [3]). Thus the roles of conductivity and resistivity become interchanged.

We are interested in the low-temperature properties of flux lines pinned to columnar defects, with filling fraction $f=N / N_{D}=\left(d / a_{0}\right)^{2}<1$, in the Bose glass phase, where all the vortices are assumed (and found) to be bound to the pinning centers. For $T$ less than a characteristic fluctuation temperature $T_{1}$, [3] one arrives at the classical limit of the corresponding boson problem $(\hbar \rightarrow 0)$, and as the flux lines are now well separated, the Bose statistics become irrelevant. Furthermore, in this limit thermal wandering is suppressed, and the flux lines will be essentially straight; hence the tilt energy in Eq. (1) can be neglected. In the boson representation we eventually have to deal with a time-independent problem defined by the two-dimensional effective Hamiltonian

$$
H=\frac{1}{2} \sum_{i \neq j}^{N_{D}} n_{i} n_{j} V\left(r_{i j}\right)+\sum_{i=1}^{N_{D}} n_{i} t_{i}
$$

where $i, j=1, \ldots, N_{D}$ denote the defect sites, randomly distributed on the $x y$ plane. $n_{i}=0,1$ is the corresponding site occupation number, and (originating in the varying pin diameters) the $t_{i}$ are random site energies, whose distribution may be chosen to be centered at $\bar{t}=0$, with width $w$ [for simplicity, we assume a flat distribution $P\left(t_{i}\right)=1 / 2 w$ for $\left|t_{i}\right| \leq w$, and $P\left(t_{i}\right)=0$ else $]$.

The Hamiltonian (2) is precisely of the form studied in the context of charge carriers localized at random impurities in doped semiconductors (Coulomb glass problem) [4, [7,8]. We remark that it is equivalent to a two-dimensional random-site, random-field antiferromagnetic Ising model with long-range exchange interactions, and has, at least to our knowledge, eluded successful analytical approaches going beyond simplifying mean-field type considerations [4], and phenomenological scaling arguments [9]. Therefore one has to resort to numerical studies using suitable Monte Carlo algorithms, as described in Refs. [4].

Basically, starting from a given distribution of $N_{D}$ defect sites, $N<N_{D}$ of which are occupied, single-particle energies are calculated according to $\epsilon_{i}=\partial H / \partial n_{i}=\sum_{j \neq i}^{N_{D}} n_{j} V\left(r_{i j}\right)+t_{i}$. The initial configuration is then relaxed by moving single "particles" to empty places, until precisely the $N$ lowest energy levels are occupied $\left(\epsilon_{\max }^{1}=\max _{n_{i}=1} \epsilon_{i}<\epsilon_{\min }^{0}=\min _{n_{i}=0} \epsilon_{i}\right)$. The 
ensuing intermediate state is then probed against all possible single-particle hops from filled to empty defect sites, with associated energy change $\Delta_{i \rightarrow j}=\epsilon_{j}-\epsilon_{i}-V\left(r_{i j}\right)$. If any $\Delta_{i \rightarrow j}<0$, the move from site $i$ to $j$ is performed, and thus the total energy is decreased. (Note that this procedure constitutes a zero-temperature algorithm.) Afterwards the "equilibration" step has to be repeated, for all the $\epsilon_{i}$ of course rearrange upon changes in the occupation numbers $n_{i}$. Finally, a chemical potential $\mu$ is calculated (approximately) by $\mu=\left(\epsilon_{\min }^{0}-\epsilon_{\max }^{1}\right) / 2$. [Formally, this corresponds to adding a term $-\mu \sum_{i} n_{i}$ to Eq. (2); this chemical potential is related to the external magnetic field $H$ by $\mu=\epsilon_{0} \ln (\lambda / \xi)-H \phi_{0} / 4 \pi-\left\langle U_{k}\right\rangle$, where $\left\langle U_{k}\right\rangle$ includes a small thermal renormalization of pinning energies.]

By repeating this procedure for different initial configurations, one may then obtain the density of states $g(\epsilon)$ from the site energy statistics. We shall use a normalization of the density of states such that $\int g(\epsilon) d \epsilon=1 / d^{2}$. To find the correct ground state for each configuration, one would in principle have to test the configuration against any simultaneous $n$-particle hops, $n=2, \ldots, \infty$. However, previous investigations have shown that terminating at $n=1$ already yields at least qualitatively reliable estimates for the energy level distributions [1, 7,8]. We have performed many such simulations for a variety of filling fractions $f$ and values of $\lambda / d$. Details of these investigations and their results shall be reported elsewhere [10]. In this Letter, we shall instead use these techniques to determine the density of states directly from experimental data, obtained as follows.

The positions of columnar defects and flux lines in a $\mathrm{Bi}_{2} \mathrm{Sr}_{2} \mathrm{CaCu}_{2} \mathrm{O}_{8}$ (BSCCO) crystal were determined simultaneously by a combined chemical etching/magnetic decoration approach (for details, see Ref. [6]). A digitized SEM image of the positions of $N=162$ flux lines and $N_{D}=686$ columnar defects (hence $f \approx 0.24$ ) for a BSCCO sample irradiated with corresponding matching field $B_{\phi}=118 \mathrm{G}(d \approx 4400 \AA)$ and decoration field $B=27 \mathrm{G}$ $\left(a_{0} \approx 9400 \AA\right)$ is shown in Fig. 1 . The critical and irreversibility temperature under these conditions are $T_{c}=87 \mathrm{~K}$ and $T_{\mathrm{irr}}=81 \mathrm{~K}$, respectively. Assuming the flux line distribution is frozen in at $T_{\mathrm{irr}}$, we estimate that the effective London penetration depth is $\lambda\left(T_{\mathrm{irr}}\right) \approx 4200 \AA$ [11], and $\lambda / d \approx 0.96$. 
In Fig. 2 the structure factor $S(q)$ for the flux lines is depicted, as obtained from $S(\mathbf{q})=$ $\frac{1}{N} \sum_{i, j}^{N} e^{i \mathbf{q}\left(\mathbf{r}_{i}-\mathbf{r}_{j}\right)}$ by averaging over directions in Fourier space (thick line). Clearly the flux line distribution is highly correlated, with $S(q)$ displaying a peak at $q_{0} a_{0} \approx 2 \pi$. We have used the experimental defect and flux line positions as an initial state for the Monte Carlo routine. In order to minimize boundary effects, we have kept the configuration fixed in a frame extending $10 \%$ (which amounts to $\approx 3 \lambda$ ) inwards from each of the rectangular boundaries. This leaves 464 sites and 106 displacable particles for the simulation. For vanishing site randomness $(w=0)$, it turns out that about $20 \%$ of the flux lines are moved in the course of the energy minimization process, which may in part be attributed to finite size effects, and also to the unaccounted variation in sample pinning energies. Using the more realistic value $w=0.1 \epsilon_{0}$ instead, we find typically $40 \%$ changes with respect to the original experimental distribution. But, as can be seen in Fig. 2, in both cases the highly correlated character of the flux line distribution is preserved, although the height of the peak at $q_{0}$ decreases upon increasing $w$. We have found, however, that substantially stronger disorder would destroy the peak in $S(q)$. Similarly, for considerably lower values of $\lambda / d(\lambda / d \leq 0.2)$, the spatial correlations also disappear, because the random site energies would then dominate over the interactions. The correlations we do find strongly support the assumption that the effective London penetration depth is the larger value $\lambda_{\text {eff }}=\lambda\left(T=T_{\text {irr }}\right)$.

The density of states for the 464 "inner" sites, as obtained from averaging over 100 runs with different assignments of random site energies, drawn though from the same distribution $P\left(t_{i}\right)$ with width $w=0.1 \epsilon_{0}$, and using $\lambda_{\text {eff }}$ for the interaction range, is shown in Fig. 3. (Results for the density of states are remarkably insensitive to the precise value $w$.) The Coulomb gap, separating the occupied and empty energy levels, is remarkably wide, its width amounting to $\approx 0.5 \epsilon_{0}$ at half maximum $\left[d^{2} g(\epsilon) \approx 0.8\right]$, i.e., almost a third of the total width of $g(\epsilon)$ there. Near its minimum, this pseudogap may be described by the formula

$$
g(\epsilon) \propto|\epsilon-\mu|^{s},
$$

with a gap exponent $s \approx 1.2$ for the specific parameter values here. In the immediate vicinity 
of the chemical potential, this power law is smeared out, and $g(\mu)$ is actually finite (but very small) due to the finite range of the interactions. We remark that in the limit $\lambda \rightarrow \infty$ and for small filling, $s \approx 3$ may be reached [10].

From this single-particle density of states, we may now infer the transport properties in the variable-range hopping regime by minimizing the free energy of two superkinks of size $R$ and separation $Z, \delta F_{\mathrm{SK}}=2 E_{K} R / d+Z \Delta(R)-f_{L} R Z$ [3]. Here, the first term consists of the energy of the double kink, with $E_{K}=\sqrt{\tilde{\epsilon}_{1} U_{0}} d$, and the third one derives from the Lorentz force $f_{L}=\phi_{0} J / c$ induced by the current $J$. The second contribution stems from the fact that for a hop of distance $R$, the available energy states lie in the interval $[\mu, \Delta(R)]$, where in $D$ dimensions (here $D=2) \Delta(R)$ is determined by the equation $\int_{\mu}^{\Delta} g(\epsilon) d \epsilon=R^{-D}$. Minimizing first for $J=0$ gives the longitudinal extent of the kink to be $Z^{*}=-2 E_{K} / d(\partial \Delta / \partial R)_{R^{*}}$. To first order in $J$, one subsequently arrives at $J \phi_{0} / c=\Delta\left(R^{*}\right) / R^{*}$, and thus $\delta F^{*}=2 E_{K} R^{*}(J) / d$ is the result for the optimized free energy. The latter finally enters the resistivity $\rho$ as an energy barrier in an activation factor, $\mathcal{E}=\rho_{0} J \exp \left(-\delta F^{*} / k_{B} T\right)$. Using $g(\epsilon)$ of Fig. 3 then amounts to studying transport slightly below the depinning temperature, at $T=77 \mathrm{~K}$, say. Note that for this field range $\tilde{\epsilon}_{1} \approx \epsilon_{0}$ and thermal renormalizations of pinning energies become relevant only for $T_{1} \approx 0.9 T_{c}(\approx 78 \mathrm{~K}$ here) [3]. Thus, all temperatures $T<T_{1}$ may be considered as "low".

In the regime where Eq. (3) holds, the final result from these considerations for the highly nonlinear current-voltage characteristics may be cast in the form

$$
\mathcal{E} \approx \rho_{0} J \exp \left[-\left(2 E_{K} / k_{B} T\right)\left(J_{0} / J\right)^{p}\right]
$$

where $p$ is an exponent generalizing Mott's law $\left[p_{0}=1 /(D+1)\right]$, which is valid in the case of vanishing interactions. For long-range interactions producing a Coulomb gap of the form (3), one finds $p=(s+1) /(D+s+1)$. Fig. 4 shows a log-log plot of the function $R^{*}(J) / d$ vs. $j=J \phi_{0} d / 2 \epsilon_{0} c$, derived from the density of states in Fig. 3 , as compared to a similarly calculated curve with the vortex repulsion being switched off. While in the latter case the result is indeed a straight line with slope $-1 / 3$, interactions considerably enhance 
the pinning by raising the effective Mott exponent to $p_{\text {eff }} \approx 0.5$ for not too low values of $J$. For $J \rightarrow 0$ the cutoff of the interaction at $\lambda$ reduces $p_{\text {eff }}$ somewhat.

Similar to the gap index $s$, from which it is derived, $p$ really should not be understood as a universal number, but rather as some effective exponent $p_{\text {eff }}$ conveniently describing the IV characteristics. Its value in general depends on both the filling $f$ and the interaction range $\lambda / d$; its maximum value $p_{\text {eff }} \approx 0.68$ is reached for $\lambda \rightarrow \infty$ and small $f$ [10. These results clearly rule out the mean-field estimate, which would yield $s=D / \sigma-1$ and $p=1 /(1+\sigma)$ for a potential $V(r) \propto r^{-\sigma}(\sigma<D$; a logarithmic interaction is recovered in the limit $\sigma \rightarrow 0$ ). Rather, they seem consistent with the analysis by Fisher, Tokuyasu, and Young, [9] who relate $p$ with the stiffness exponent $\Theta \approx-0.5$ and a fractal index $1 \leq \psi_{Q} \leq 2$ for the supposedly equivalent gauge glass model. Their scaling relation $p=1 /\left(1+|\Theta| / \psi_{Q}\right)$, indeed yields $p \approx 2 / 3$ for $\psi_{Q} \approx 1$. It is, however, not yet settled if universality does apply in the purely long-range limit, and even the results by Möbius and Richter, [8] who were able to simulate the $1 / r$ Coulomb problem in two and three dimensions for very large systems, have apparently not reached the fully asymptotic regime. Also, our derivation of $R^{*}(J)$ from $g(\epsilon)$ of course constitutes an approximation which neglects some subtle correlations, e.g. the spatial clustering of those sites which are energetically close to $\mu$, [7,[1]], and may be subject to corrections in the limit $J \rightarrow 0$.

In summary, we have demonstrated that the vortex-vortex repulsion can lead to remarkable correlations both in real space and in the single-particle density of states, even for $\lambda \approx a_{0}$, which is easily accessible in experiment. An important consequence of these correlation effects is the drastic enhancement of flux line pinning to columnar defects in the Bose glass phase, whenever $\lambda \geq a_{0}$.

We benefitted from discussions with A.L. Efros, D.S. Fisher, T. Hwa, P. Le Doussal, and V.M. Vinokur. This work was supported by the National Science Foundation, primarily by the MRSEC Program through Grant DMR-9400396, and through Grants DMR-9417047 and DMR-9306684. U.C.T. acknowledges support from the Deutsche Forschungsgemeinschaft (DFG) under Contract Ta. 177/1-1. 


\section{REFERENCES}

[1] For a recent review, see G. Blatter, M.V. Feigel'man, V.B. Geshkenbein, A.I. Larkin, and V.M. Vinokur, Rev. Mod. Phys. 66, 1125 (1994).

[2] See, e.g., L. Civale et al., Phys. Rev. Lett. 67, 648 (1991); M. Konczykowski et al., Phys. Rev. B 44, 7167 (1991); R.C. Budhani, M. Suenaga, and S.H. Liou, Phys. Lett. 69, 3816 (1992).

[3] D.R. Nelson and V.M. Vinokur, Phys. Rev. B 48, 13060 (1993); and references therein.

[4] See, e.g., B.I. Shklovskii and A.L. Efros, Electronic Properties of Doped Semiconductors (Springer, New York, 1984); and references therein.

[5] S. Behler et al., Phys. Rev. Lett. 72, 1750 (1994); Z. Phys. B 94, 213 (1994).

[6] H. Dai, S. Yoon, J. Liu, R.C. Budhani, and C.M. Lieber, Science 265, 1552 (1994); and references therein.

[7] J.H. Davies, P.A. Lee, and T.M. Rice, Phys. Rev. Lett. 49, 758 (1982); Phys. Rev. B 29, 4260 (1984); E.I. Levin, V.L. Nguen, B.I. Shklovskii, and A.L. Efros, Sov. Phys. JETP 65, 842 (1987) [Zh. Eksp. Teor. Fiz. 92, 1499 (1987)].

[8] A. Möbius, M. Richter, and B. Drittler, Phys. Rev. B 45, 11568 (1992).

[9] M.P.A. Fisher, T.A. Tokuyasu, and A.P. Young, Phys. Rev. Lett. 66, 2931 (1991).

[10] U.C. Täuber and D.R. Nelson, unpublished.

[11] Upon assusing $\lambda_{0}=\lambda(T=0) \approx 2100 \AA$ and the two-fluid formula, $\lambda(T)=\lambda_{0} /[1-$ $\left.\left(T / T_{c}\right)^{4}\right]^{1 / 2}$, we find that $\lambda\left(T_{\mathrm{irr}}\right) \approx 2 \lambda_{0}$. 


\section{FIGURES}

FIG. 1. Positions of columnar pins (open circles) and flux lines (filled circles), as obtained from the experiment.

FIG. 2. Flux line structure function $S(q)$ as obtained from the experiment (thick line), and from the simulation with $w=0$ (dashed), and $w=0.1 \epsilon_{0}$ (thin line), averaged over 100 different assignments of random site energies.

FIG. 3. Normalized density of states $G(E)=d^{2} g(\epsilon)$ as function of the single-particle

energies $E=\epsilon / 2 \epsilon_{0}$, averaged over 100 runs $\left(\lambda / d=0.96, w=0.1 \epsilon_{0}\right)$. The mean chemical potential is $\mu / 2 \epsilon_{0}=0.65$.

FIG. 4. Double-logarithmic plot of the exponential factor $R^{*}(J) / d$ for Mott variablerange hopping vs. $j=J \phi_{0} d / 2 \epsilon_{0} c$, as obtained from the density of states in Fig.3 (filled circles), compared to the result of the non-interacting case (open circles). 
Figure 1

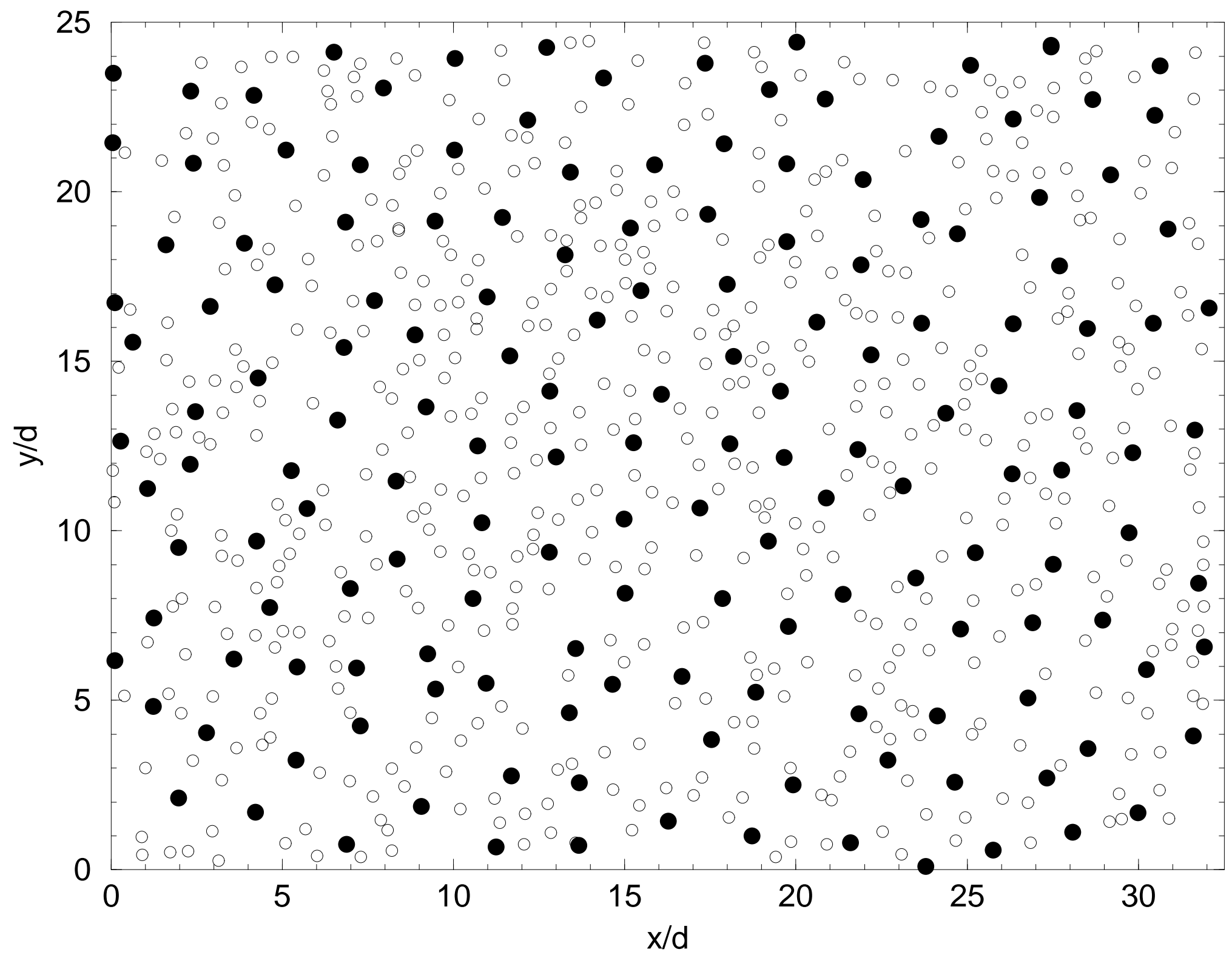


Figure 2

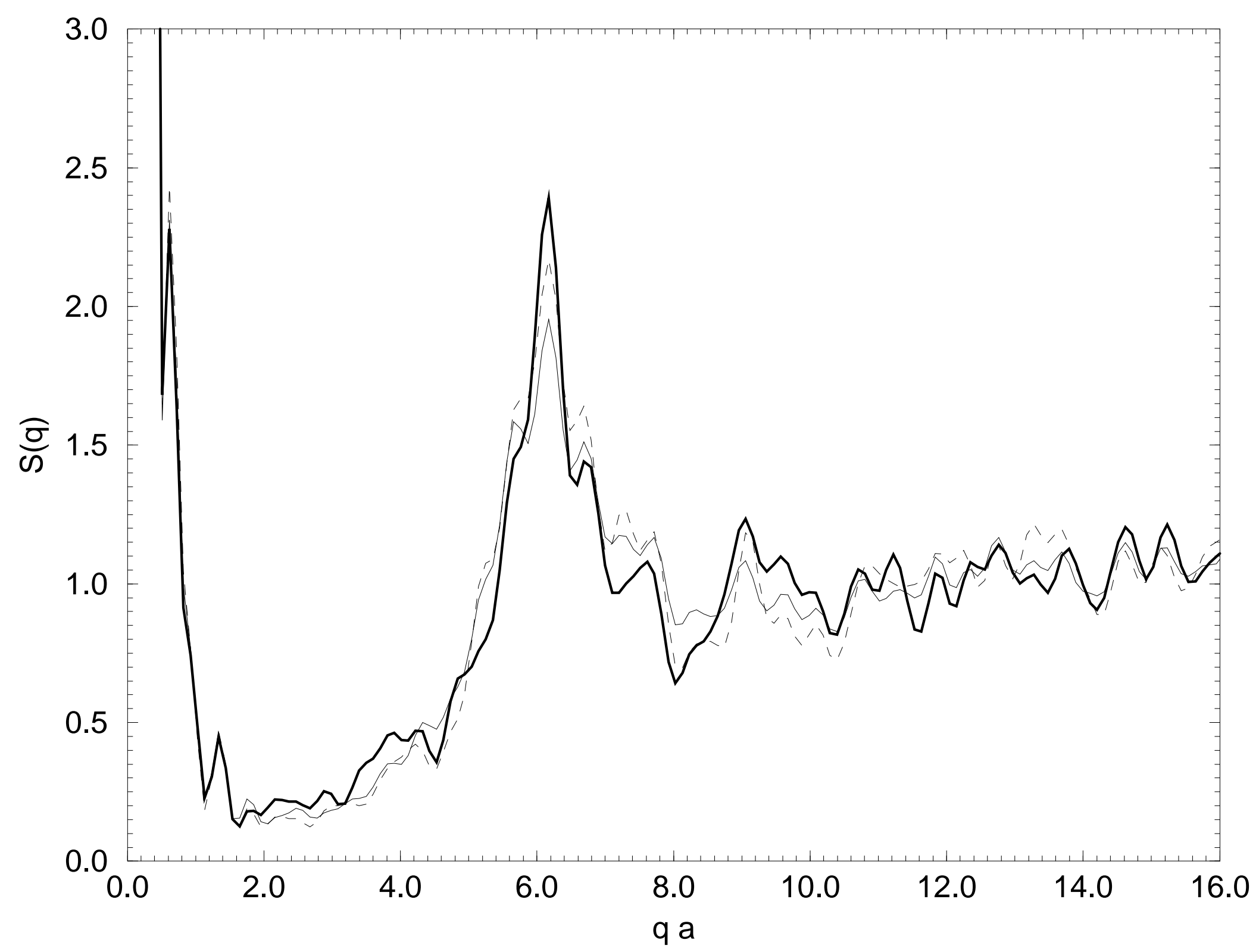


Figure 3

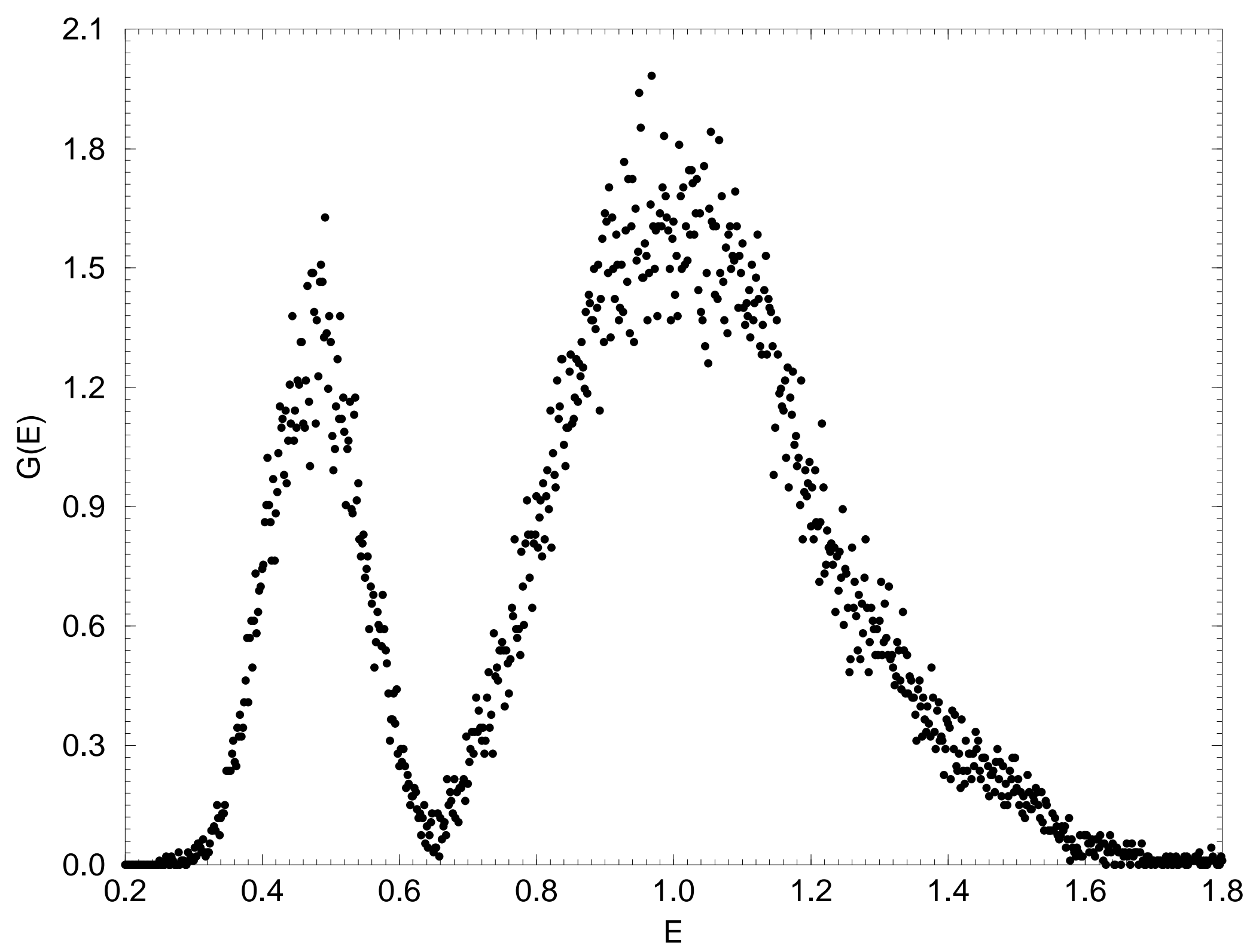


Figure 4

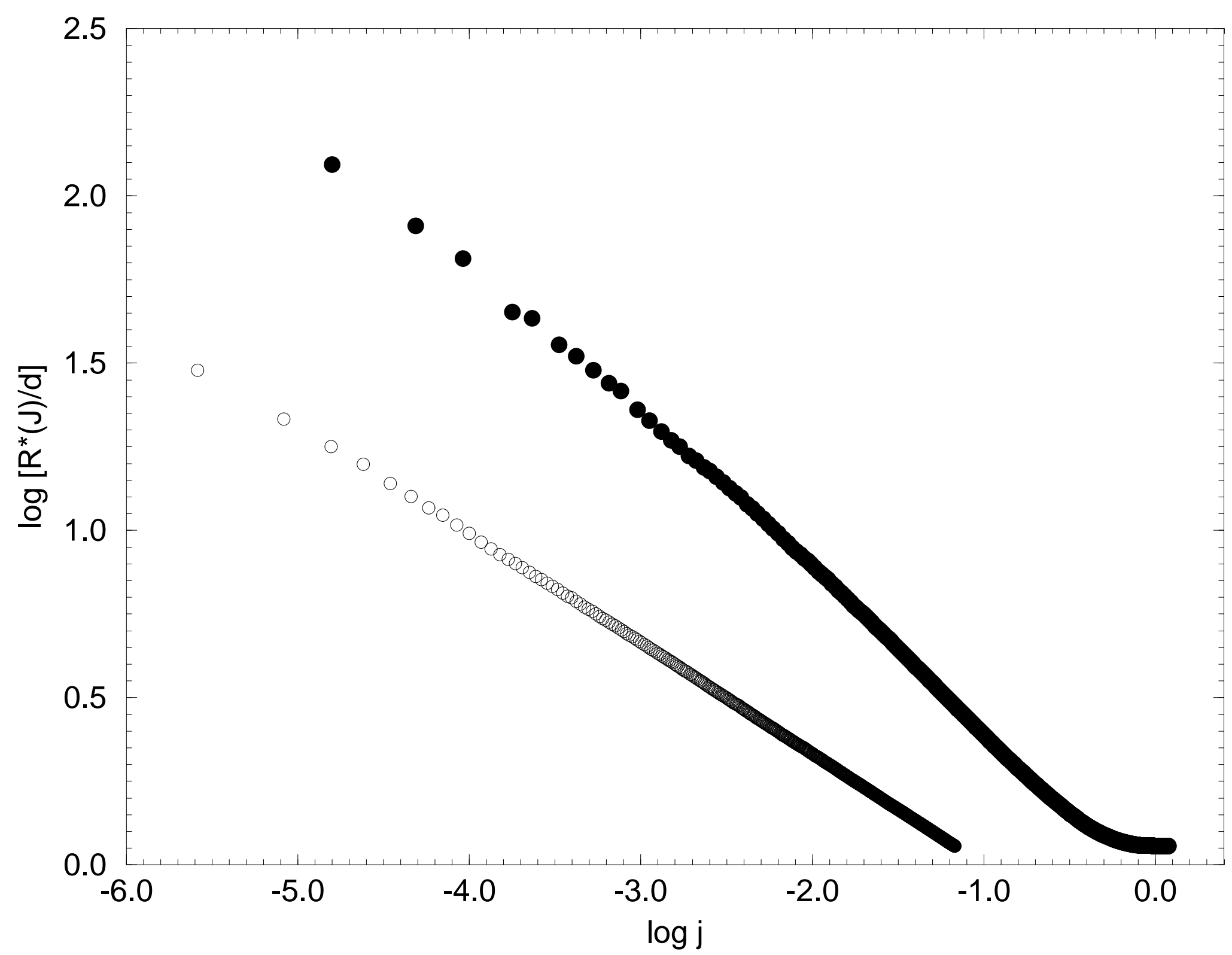

